\title{
Attitude Stabilization of the PMM Satellite Using a LQG-Based Control Strategy
}

A.C. SANTANA ${ }^{1}$, Programa de Pós-Graduação em Engenharia da Informação, Universidade Federal do ABC, Rua Catequese, 242, Bairro Jardim, 09090-400 Santo André, SP, Brasil.

L.S. MARTINS-FILHO ${ }^{2}$, Centro de Engenharia, Modelagem e Ciências Sociais Aplicadas, Universidade Federal do ABC, Rua Catequese, 242, Bairro Jardim, 09090-400 Santo André, SP, Brasil.

G. ARANTES JR. ${ }^{3}$, Zentrum für angewandte Raumfahrttechnologie und Mikrogravitation, Universität Bremen, Am Fallturm, D-28359 Bremen, Germany.

\begin{abstract}
This paper deals with the problem of 3-axis attitude stabilization of a satellite subjected to dynamics perturbations. The controller is based on the Linear Quadratic Gaussian control theory (LQG). The main purpose of the work is to synthesize a control law characterized by simplicity on implementation. The study considers the specific case of the Brazilian satellite PMM. The article presents the system modelling, the proposed control, and analysis of simulation results.
\end{abstract}

Keywords. Satellite attitude control, Three-axis attitude stabilization, Thruster actuators, LQG control theory.

\section{Introduction}

An important problem of the artificial satellites technology is the precise control of the spacecraft orientation in a known reference frame. In fact, the success of different missions depends of the precision on the spacecraft attitude, ensuring the adequate performance of the payload equipments and instruments in terms of pointing precision. That is the case of applications in remote sensing, meteorology, communication, and astrophysics as well.

Though diverse solutions for this classic problem has been proposed since the beginning of the space engineering, this functional aspect of satellites stays as an interesting subject of studies. The main motivation is the search of a well adapted solution for each specific satellite, considering the peculiar features, and combining efficiency and robustness with implementation simplicity. In our precedent work,

\footnotetext{
1 adrielle.santana@ufabc.edu.br

${ }^{2}$ luiz.martins@ufabc.edu.br

3 gilberto@spacetec.zarm.uni-bremen.de
} 
we have proposed a linear control based on LQR theory [2]. In other examples of studies, we find variations of the PID controller, e.g. in Show et al. [8]. Application of nonlinear control techniques for a critical case, when a pair of attitude actuators fails, is proposed by Tsiotras et al. [9]. Yang and Kung [10] propose a control approach using a nonlinear $H_{\infty}$ controller; while in $\mathrm{Wu}$ and Chen [13] a mixed $H_{2}$ / $H_{\infty}$ approach is investigated.

In this work, we consider the case of a satellite that has been developed in the context of Brazilian Space Program [1], the PMM satellite (Multi-Mission Platform) designed for different applications and missions (see Fig. 1). We propose a strategy of spacecraft stabilization using three pairs of two-sides thrusters acting to provide the computed control torques, and adopting a Linear Quadratic Gaussian (LQG) control approach. The main purpose of this study is to contribute to the PMM satellite project. We suppose that the problem of attitude determination is satisfactorily solved, including all theoretical and practical aspects of computing procedure for the estimation of satellite orientation. The proposed approach was tested through a series of numerical simulations considering the complete non-linear satellite attitude model, and the control module computing the thruster force.

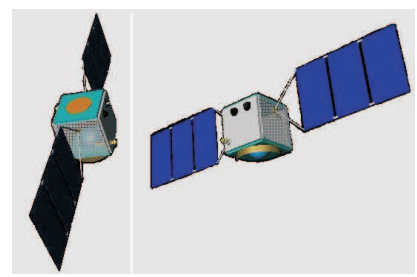

Figure 1: The graphic interface of the simulation tool built in virtual reality modelling language (VRML) showing the satellite model without payload.

The paper is organized as follows: the problem formulation is presented in Section 2, including the aspects of attitude representation and the kinematics and dynamics modeling. Besides that, the adopted control strategy is discussed in Section 3. Then, a description of the validation tests and numerical simulations, as well as the presentation and analysis of their results, are shown in Section 4 . The final remarks and the perspective of future works conclude the paper, Section 5.

\section{2. $\quad$ Satellite Attitude Modelling}

In this section we describe the mathematical model of the satellite attitude, including the kinematics and the dynamics, and the linearization of the satellite model to allow the application of a linear control approach.

The satellite attitude will be defined in this work by the position of the three principal axes of inertia with respect to the Local-Vertical-Local-Horizontal (LVLH) frame [11]. Naming $R$ the direction cosine matrix between the LHLV frame and the reference frame fastened to the satellite body. During the stabilization mode only small angular variations are considered, then the Euler angles parametrization is an 
appropriate choice as it guarantees non-singularity. Thus, by using Euler angles in an asymmetric sequence $3-2-1$ for describing a rotation matrix $[4,11]$, one finds:

$$
R=R_{x}(\phi) R_{y}(\theta) R_{z}(\psi)=\left[\begin{array}{ccc}
c \psi c \theta & s \psi c \theta & -s \theta \\
-c \phi s \psi+s \phi s \theta c \psi & c \phi c \psi+s \phi s \theta s \psi & s \phi c \theta \\
s \phi s \psi+c \phi s \theta c \psi & -s \phi c \psi+c \phi s \theta s \psi & c \phi c \theta
\end{array}\right]
$$

The kinematics equation for the satellite attitude is given by:

$$
\dot{R}(t)=S(\boldsymbol{\omega}(t)) R(t)
$$

where $S$ is the skew-symmetric operator with respect to angular velocity $\boldsymbol{\omega}$, given by:

$$
S(\boldsymbol{\omega})=\left[\begin{array}{ccc}
0 & -\omega_{z} & \omega_{y} \\
\omega_{z} & 0 & -\omega_{x} \\
-\omega_{y} & \omega_{x} & 0
\end{array}\right]
$$

For the rotation sequence $3-2-1$, the satellite attitude kinematics can be described as follows $[11,12]$ :

$$
\left[\begin{array}{c}
\dot{\phi} \\
\dot{\theta} \\
\dot{\psi}
\end{array}\right]=\frac{1}{c \theta}\left[\begin{array}{ccc}
c \theta & s \phi s \theta & c \phi s \theta \\
0 & c \phi c \theta & -s \phi c \theta \\
0 & s \phi & c \phi
\end{array}\right] \boldsymbol{\omega}_{i b}^{b}+\frac{\omega_{0}}{\cos \theta}\left[\begin{array}{c}
s \psi \\
c \theta c \psi \\
s \theta s \psi
\end{array}\right]
$$

where $\omega_{0}$ is the mean orbital motion and $\boldsymbol{\omega}_{i b}^{b}$ is the satellite angular velocity vector with respect to the inertial reference frame, expressed in the reference frame fastened to the satellite body. The linearized kinematic equation is given by:

$$
\boldsymbol{\omega}_{i b}^{b}=\left[\begin{array}{c}
\dot{\phi} \\
\dot{\theta} \\
\dot{\psi}
\end{array}\right]+\omega_{0}\left[\begin{array}{c}
-\psi \\
-1 \\
\phi
\end{array}\right]
$$

The dynamic of a satellite attitude, equipped with six two-sides thrusters is modelled by using the Euler equations. Furthermore, the attitude dynamic is written with respect to the reference frame fastened to the satellite body, as follows:

$$
\boldsymbol{\tau}_{e x t}=\left[\frac{d \mathbf{h}}{d t}\right]_{b}+\boldsymbol{\omega}_{i b}^{b} \times \mathbf{h}_{b}
$$

where $\mathbf{h}_{b}=J \boldsymbol{\omega}_{i b}^{b}$ and $\dot{\mathbf{h}}_{b}=\left[\frac{d \mathbf{h}}{d t}\right]_{b}=J \dot{\boldsymbol{\omega}}_{i b}^{b}$. In these equations, $J$ is the satellite inertia matrix and $\boldsymbol{\tau}_{\text {ext }}$ are the external torques acting in the system, i.e. perturbations and thruster actuation. Rewriting the Eq. 2.6, we have:

$$
J \dot{\boldsymbol{\omega}}_{i b}^{b}+S\left(\boldsymbol{\omega}_{i b}^{b}\right) J \boldsymbol{\omega}_{i b}^{b}=\boldsymbol{\tau}_{d}^{b}+\boldsymbol{\tau}_{p}^{b}
$$

where $\tau_{d}^{b}$ represents all the disturbance torques, e.g. atmosphere drag, and $\tau_{p}^{b}$ represents the control torques. The assumed thruster configuration is based on a 
simple symmetric geometric distribution of those six two-side thruster. Each twoside thruster is placed in the center of each side of the satellite. In this case, the control torques are given by:

$$
\begin{aligned}
\tau_{\text {roll }}^{b} & =l u_{1}+l u_{2} \\
\tau_{\text {pitch }}^{b} & =l u_{3}+l u_{4} \\
\tau_{\text {yaw }}^{b} & =l u_{5}+l u_{6}
\end{aligned}
$$

where $u_{i},(i=1, \ldots, 6)$ is the control command and $l$ is the distance from the mass center to each two-side thruster. The Eq. 2.8 shows that there is one thruster pair for each principal axis of inertia of the satellite body. The value of the pulse-mode thruster, considering the model discussed in Salles et al. [6], is $5 \mathrm{~N}$.

In the dynamic model the gravity gradient torque is considered. It is caused for the difference in the intensity and direction of the gravitational force acting in different parts of the satellite [7]. Due to the physical characteristic of PMM, the gravity gradient torque is very small and for a final desired attitude in a specific case of Earth pointing applications, it acts as a collaborative disturbance. For small angle maneuvers, we can use the model of the gravity gradient torque given by $[12,4]$ :

$$
\boldsymbol{\tau}_{g}^{b}=3 \omega_{0}^{2}\left[\begin{array}{c}
\left(J_{z}-J_{y}\right) \phi \\
\left(J_{x}-J_{z}\right) \theta \\
0
\end{array}\right]
$$

By using the linearized kinematics Eq. 2.5, replacing in the Eq. 2.7, and including the control torques Eq. 2.8, and the gravity gradient torque Eq. 2.9, we obtain the following dynamic equations:

$$
\begin{aligned}
J_{x} \ddot{\phi}= & \phi\left[4 \omega_{0}^{2}\left(J_{z}-J_{y}\right)-\omega_{0} \dot{\theta}\left(J_{z}-J_{y}\right)\right]+\dot{\theta} \dot{\psi}\left(J_{y}-J_{z}\right) \\
& +\psi \omega_{0}\left(J_{x}-J_{y}+J_{z}\right)+l u_{1}+l u_{2} \\
J_{y} \ddot{\theta}= & 3 \omega_{0}^{2}\left(J_{x}-J_{z}\right) \theta+\phi\left[\psi \omega_{0}^{2}\left(J_{x}-J_{z}\right)+\dot{\phi} \omega_{0}\left(J_{z}-J_{x}\right)\right] \\
& +\dot{\psi} \psi \omega_{0}\left(J_{x}-J_{z}\right)+\dot{\psi} \dot{\phi}\left(J_{z}-J_{x}\right)+l u_{3}+l u_{4} \\
J_{z} \ddot{\psi}= & \psi\left[\omega_{0}^{2}\left(J_{x}-J_{y}\right)+\dot{\theta} \omega_{0}\left(J_{y}-J_{x}\right)\right] \\
& +\dot{\phi}\left[\omega_{0}\left(J_{y}-J_{x}-J_{z}\right)+\dot{\theta}\left(J_{x}-J_{y}\right)\right]+l u_{5}+l u_{6}
\end{aligned}
$$

The state equation for this system is obtained from the linearization of Eq. 2.10, given by:

$$
\dot{\mathbf{x}}=A \mathbf{x}+B \mathbf{u}
$$

where $\mathbf{x}=[\phi, \theta, \psi, \dot{\phi}, \dot{\theta}, \dot{\psi}]^{T}, \mathbf{x}=\left[u_{1}, \cdots, u_{6}\right]^{T}$, and the matrices $A$ and $B$ are given by: 


$$
\begin{aligned}
& A=\left[\begin{array}{cccccc}
0 & 0 & 0 & 1 & 0 & 0 \\
0 & 0 & 0 & 0 & 1 & 0 \\
0 & 0 & 0 & 0 & 0 & 1 \\
\frac{4 \omega_{0}^{2}\left(J_{z}-J_{y}\right)}{J_{x}} & 0 & 0 & 0 & 0 & \frac{\omega_{0}\left(J_{x}-J_{y}+J_{z}\right)}{J_{x}} \\
0 & \frac{3 \omega_{0}^{2}\left(J_{x}-J_{z}\right)}{J_{y}} & 0 & 0 & 0 & 0 \\
0 & 0 & \frac{\omega_{0}^{2}\left(J_{x}-J_{y}\right)}{J_{z}} & \frac{\omega_{0}\left(J_{y}-J_{x}-J_{z}\right)}{J_{z}} & 0 & 0
\end{array}\right] \\
& B=\left[\begin{array}{cccccc}
0 & 0 & 0 & 0 & 0 & 0 \\
0 & 0 & 0 & 0 & 0 & 0 \\
0 & 0 & 0 & 0 & 0 & 0 \\
\frac{l}{J_{x}} & \frac{l}{J_{x}} & 0 & 0 & 0 & 0 \\
0 & 0 & \frac{l}{J_{y}} & \frac{l}{J_{y}} & 0 & 0 \\
0 & 0 & 0 & 0 & \frac{l}{J_{z}} & \frac{l}{J_{z}}
\end{array}\right]
\end{aligned}
$$

This linearized system is used on the control law synthesis, while the strict model of attitude dynamics, a nonlinear system, is used in the satellite simulation.

\section{The LQG Controller Design}

The Linear Quadratic Gaussian (LQG) control consist of a technique for designing optimal dynamic regulators, based on the state-space system modelling. This technique is based on the search of the trade off between regulation performance and control efforts, and it takes into account process disturbances and measurement noises. Basically, the LQG approach addresses the problem where we consider a system dynamic model perturbed by a dynamical noise $\mathbf{w}$, and a state observation corrupted by measurement noise $\boldsymbol{\nu}$, affecting the sensors data acquisition.

The LQG regulator comprises an optimal state-feedback gain and a Kalman filter state estimator. This technique requires a linear or linearized state-space model of the system with the addition of the noise effect as shown in Eq. 3.1. The dynamic model is given by:

$$
\begin{aligned}
\dot{\mathbf{x}} & =A \mathbf{x}+B \mathbf{u}+G \mathbf{w} \\
\dot{\mathbf{y}}_{\nu} & =C \mathbf{x}+D \mathbf{u}+\boldsymbol{\nu}
\end{aligned}
$$

where $\dot{\mathbf{y}}_{\nu}$ is the measurement that results from known inputs (state and control variables) and is corrupted by the noise inputs $\mathbf{w}$ and $\dot{\mathbf{y}}_{\nu}$. The process and measurements noises are modelled as white noises.

The LQG design consist of obtaining the feedback control law in the form $\mathbf{u}=-K \widehat{\mathbf{x}}$, which optimizes the regulation index given by a quadratic performance criterion:

$$
J=\int_{0}^{\infty}\left(\mathbf{x}^{T} Q \mathbf{x}+\mathbf{u}^{T} R \mathbf{u}\right) d t
$$


where $Q$, and $R$ are weighting matrices that define the tradeoff between regulation performance and control efforts, i.e. the relative weight of how fast the state $\mathbf{x}(t)$ goes to zero and the magnitude of the control efforts $\mathbf{u}$.

Considering a time invariant system, the gain matrix $K$ is obtained by solving the algebraic Riccati equation (Eq.3.3) and taking $K=R^{-1} B^{T} P$.

$$
A^{T} P+P A-P B R^{-1} B^{T} P+Q=0
$$

The Kalman filter is used as observer to obtain the estimation of the state variable, i.e. $\widehat{\mathbf{x}}$. The filter equation is given by:

$$
\dot{\hat{\mathbf{x}}}=A \widehat{\mathbf{x}}+B \mathbf{u}+L\left(\mathbf{y}_{\nu}-C \widehat{\mathbf{x}}-D \mathbf{u}\right)
$$

where $L$ is the Kalman gain. This filter is optimal when the disturbances affecting the process dynamics and the measurement are modelled as Gaussian white noises. The block diagram can now includes the Kalman filter, and the schematic description of the regulation problem is shown in Fig. 2.

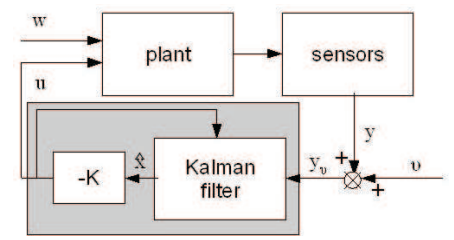

Figure 2: The general scheme of the LQG regulation including the Kalman filter.

\section{Numerical Simulations and Results}

The proposed attitude stabilization strategy, based on the LQG regulation, was tested by using numerical simulations. Although several simulations over a wide range of initial condition for attitude were performed, just one case is shown. The parameters and specifications considered in these simulations are shown in Tab. 1,

Table 1: Simulation parameters

\begin{tabular}{|c|c|}
\hline Parameters & Values \\
\hline $\begin{array}{c}\text { Principal momentum } \\
\text { of inertia (without payload) } \\
\left(\mathrm{Kg} \mathrm{m}^{2}\right)\end{array}$ & $\begin{array}{c}J_{x}=305.89126 \\
J_{y}=314.06488 \\
J_{z}=167.33919\end{array}$ \\
\hline Torque arm $(\mathrm{m})$ & $l=0.5$ \\
\hline Mean orbital motion $(\mathrm{rad} / \mathrm{s})$ & $\omega_{0}=0.001$ \\
\hline Mass $(\mathrm{Kg})$ & 578.05239 \\
\hline Height $(\mathrm{Km})$ & 750 \\
\hline Maximum force $(\mathrm{N})$ & 5 \\
\hline Eccentricity & $\cong 0$ \\
\hline Initial attitude (degrees) & $(\phi, \theta, \psi)=(10,10,10)$ \\
\hline Initial Angular Rate (degrees/s) & $\boldsymbol{\omega}_{i b}^{b}=[1,1,1]^{T}$ \\
\hline
\end{tabular}

including the values of principal momentum of inertia (without payload) [1]. The control tuning matrices $R$ and $Q$ were obtained through iterative process following 

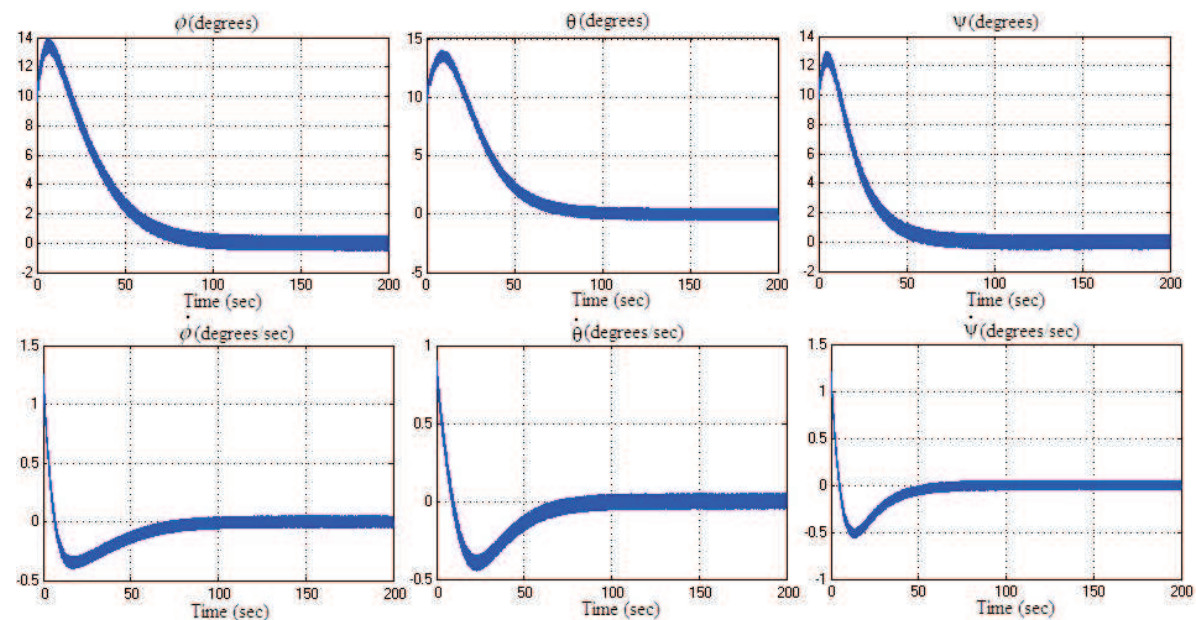

Figure 3: The results of numerical simulations: actual values of angles and angular velocities during the stabilization of the satellite attitude.

expectable requirements, e.g. allowed (non-saturation) control effort and reasonable stabilization time.

The designed controller, presented in Section 3., is applied to the nonlinear model of the satellite. The time variation of state variables (Euler angles), and the respective rates as sensing for the sensors, e.g. star sensor and gyros, on the realized simulation are shown in Fig. 3.

The time variation of the estimated state by the Kalman filter are shown in Fig. 4. This result shows very clear the effect of the filter, the noisy signal Fig. 3 from the sensors are filtered and smoothed by the filter. Additionally, the controller is fed by the estimated state. Fig. 5 shows the control effort which act in the system.

These figures show the stabilization of the satellite attitude in a reasonable time, i.e. the attitude and angular velocities go to the desired values in around one half minutes for row and pitch and one minute for yaw. Furthermore, though the control torques reach their upper limit in the beginning of the maneuver but promptly fall to low values when the attitude moves to the commanded orientation.

The obtained results, using a refined dynamic model (non-linear) and physical parameters of the PMM satellite, allow us to conclude, positively, about the proposed control strategy based on the Linear Quadratic Gaussian Theory. In spite of the approach simplicity of the linear control applied to a highly nonlinear system, the results are pointing to the adequacy of the application of this strategy to this problem, in particular for the studied stabilization mode. Besides, the presented approach presents attractive advantages for the controller implementation, i.e. optimality in the square sense, guarantee of stability margins and easily control design. 

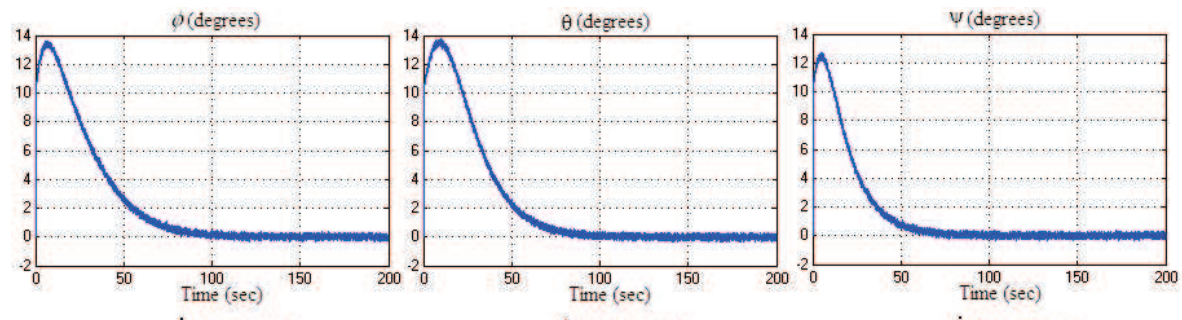

$\dot{o}$ (degrees sec)
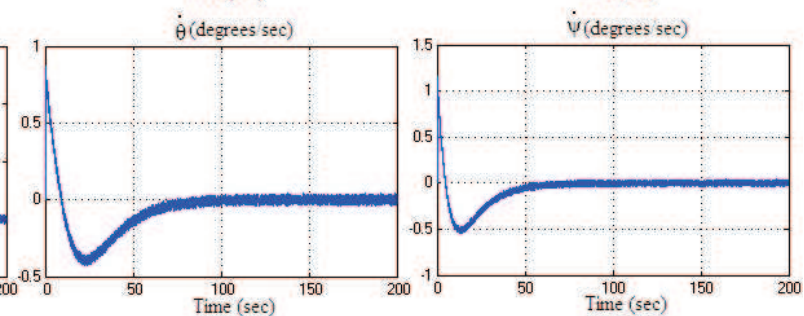

Figure 4: The results of numerical simulations: estimated values of angles and angular velocities during the stabilization of the satellite attitude.
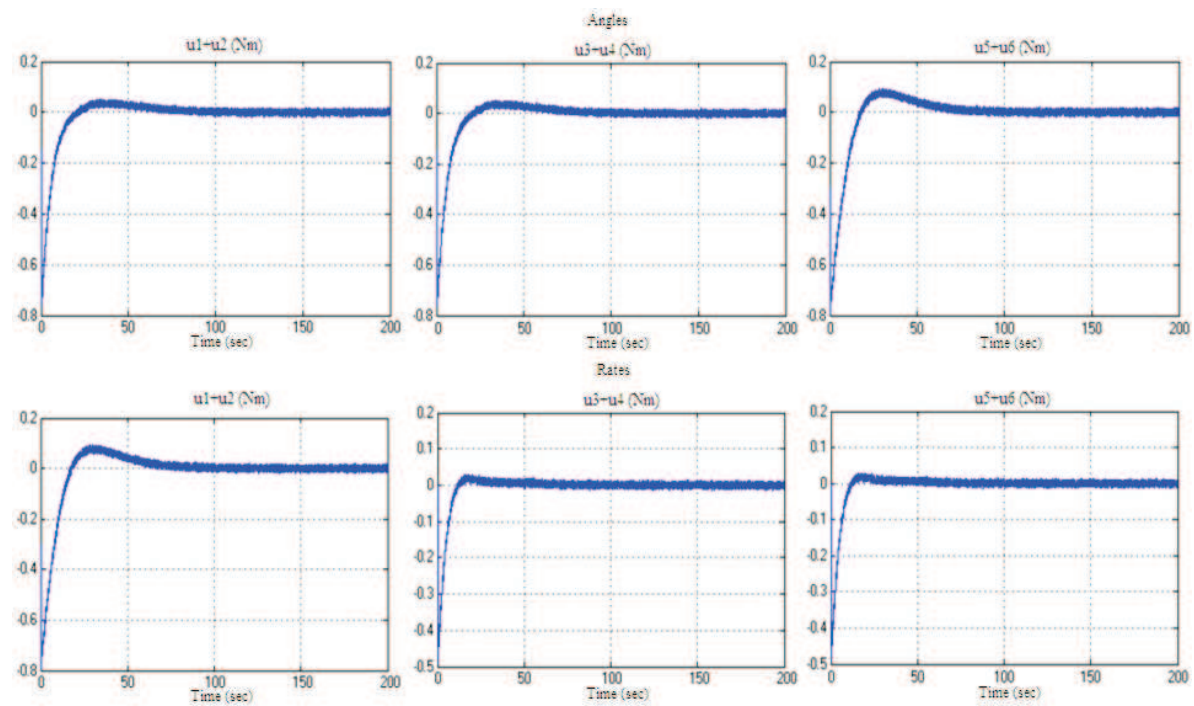

Figure 5: The results of numerical simulations: values of control torques provided by the actuators during the stabilization of the satellite attitude. 


\section{Conclusion}

The obtained numerical simulations results lead to the conclusion that the control strategy based on the LQG theory stabilizes the system exponentially. This is an interesting result for the reason that the problem of satellite attitude stabilization constitutes a highly nonlinear control system problem and the application of linear approaches represents an attractive issue in terms of real-time implementation. This study on attitude control aims to contribute to the PMM project, the Brazilian standard satellite conceived to be used on a large number and different types of missions, in the context of an ever-advancing Brazilian space program.

The perspectives of future works include the analyze for others control attitude modes, e.g. on failure mode (i.e. when the satellite can use only two pairs of twosides thrusters), and acquisition mode by adding the LQ tracking approach. In a further study the actuator model and a more appropriated thruster configuration matrix should be taken into consideration as well as other systems perturbations (e.g. atmosphere drag, J2 effect, etc). Additionally, a analysis of the condition of the stabilization due to non-linear nature of the problem may be made.

\section{Acknowledgement.}

The authors acknowledge the support of Conselho Nacional de Desenvolvimento Científico e Tecnológico - CNPq, Fundação de Amparo à Pesquisa do Estado de S. Paulo - FAPESP, and Coordenação de Aperfeiçoamento de Pessoal de Nível Superior - CAPES.

Resumo. Esse trabalho trata do problema de estabilização em 3 eixos de um satélite sujeito à perturbações dinâmicas. $\mathrm{O}$ controlador se baseia na teoria de controle linear quadrático gaussiano (LQG). O objetivo principal é sintetizar uma lei de controle caracterizada pela simplicidade de implantação. O estudo considera o caso específico do satélite brasileiro PMM O artigo apresenta a modelagem do sistema, o controle proposto, e a análise dos resultados de simulação numérica.

\section{References}

[1] AEB - Agência Espacial Brasileira, "National Space Activities Program PNAE 1998-2007", Brazilian Space Agency Report, AEB, Brasilia, 1998.

[2] G. Arantes Jr., A.C. Santana, L.S. Martins-Filho, Dynamics and control of three-axis satellites by thruster actuators using a linear quadratic regulator, in "Proc. of International Congress of Mechanical Science", Brasilia, pp. 1-9, 2007.

[3] C.A.P. Dorato, V. Cerone, "Linear Quadratic Control: an Introduction", Prentice Hall, Englewood Cliffs, 1995.

[4] M.H. Kaplan, "Modern Spacecraft Dynamics and Control", John Wiley and Sons, New York, 1976. 
[5] J.M. Maciejowski, "Multivariable Feedback Design", Addison Wesley Publishing, New York, 1989.

[6] C.E.R. Salles et al., Sistemas propulsivos para satélites: desenvolvimento e Qualificação,in "Anais do Encontro para a Qualidade de Laboratórios", São Paulo, pp. 1-8, 2005.

[7] L.T.F. Sene, V. Orlando, M.C. Zanardi, "Propagação da Atitude de Satélites Artificiais com Quatérnions incluindo Torques Magnéticos e Torque Gradiente de Gravidade", Technical Report INPE-14020-PRE/9195, INPE, S. José dos Campos, Brazil, 2006.

[8] L.L. Show, J.C Juang, C.T Lin, Y.W. Jan, Spacecraft robust attitude tracking design: PID control approach, in "Proc. of the American Control Conference", Anchorage, pp. 1360-1365, 2002.

[9] P. Tsiotras, M. Corless, J.M. Longuski, A nouvel approach to the attitude control of axisymmetric spacecraft, Automatica, 31, No. 8 (1995), 1099-1112.

[10] C.D. Yang, C.C. Kung, Nonlinear $H_{\infty}$ flight control of general six degreeof-freedom motions, Journal of Guidance Control and Dynamics, 23, No. 2 (2000), 278-288.

[11] J.R. Wertz (Ed.), "Spacecraft Attitude Determination and Control", Reidel, Dordrecht, 1978.

[12] H.W.B. Wie, A. Arapostathis, Quaternion Feedback Regulator for Spacecraft Eigenaxis Rotations, Journal of Guidance Control and Dynamics, 12, No. 3 (1989), 375-380.

[13] C.S. Wu, B.S. Chen, Unified design for $H_{2}, H_{\infty}$ and mixed control of spacecraft, Journal of Guidance Control and Dynamics, 22, No. 6 (1999), 884-896. 